\title{
Diffusive Thermal Dynamics for the Ising Ferromagnet
}

\author{
P. Buonsante ${ }^{1,2,3, *}$, R. Burioni ${ }^{1,2, \dagger}$, D. Cassi ${ }^{1,2, \ddagger}$ and A. Vezzani ${ }^{1,2, \S}$ \\ 1 Istituto Nazionale Fisica della Materia. \\ 2 Dipartimento di Fisica, \\ Università degli Studi di Parma, \\ Parco Area delle Scienze 7/a - 43100 Parma, Italy. \\ 3 Dipartimento di Fisica, Politecnico di Torino, \\ Corso Duca degli Abruzzi 24, 10129 - Torino, Italy.
}

(Dated: November 21, 2018)

\begin{abstract}
We introduce a thermal dynamics for the Ising ferromagnet where the energy variations occurring within the system exhibit a diffusive character typical of thermalizing agents such as e.g. localized excitations. Time evolution is provided by a walker hopping across the sites of the underlying lattice according to local probabilities depending on the usual Boltzmann weight at a given temperature. Despite the canonical hopping probabilities the walker drives the system to a stationary state which is not reducible to the canonical equilibrium state in a trivial way. The system still exhibits a magnetic phase transition occurring at a finite value of the temperature larger than the canonical one. The dependence of the model on the density of walkers realizing the dynamics is also discussed. Interestingly the differences between the stationary state and the Boltzmann equilibrium state decrease with increasing number of walkers.
\end{abstract}

PACS Numbers: 2.70.Uu, 2.70.Tt, 5.50.+q, 5.10.Ln

\section{INTRODUCTION AND MOTIVATIONS}

Consider an Ising ferromagnet consisting of an assembly of $N$ spins, each placed at a site of a $d$-dimensional Euclidean lattice. Let lowercase italic letters, $i, j, \cdots$, denote such sites, and $s_{i}, s_{j}, \cdots$, the relevant spin variables, so that the energy pertaining to a given configuration $\mathbf{s}=\left\{s_{i}\right\}_{i=1}^{N}$ has the form

$$
E(\mathbf{s})=-\frac{1}{2} \sum_{i=1}^{N} s_{i} \sum_{j \sim i} s_{j},
$$

where the symbol $\sim$ in the second sum restricts it to the sites $j$ adjacent to $i$.

As it is well known, the Ising modell is amenable of an exact solution on simple two dimensional lattices䏣, whereas for more complex lattices or higher dimensions the study of its thermodynamic properties strongly relies on numeric simulations. According to the so-called $d y$ namic Monte Carlo method, the thermodynamic canonical average of a generic observable $X(\mathbf{s})$ is obtained as a simple algebraic average over the Markov chain of configurations produced by a suitable stochastic algorithm 1 . Indeed each configuration is obtained from the previous one so that, in the asymptotic limit, the probability $P_{T}(\mathbf{s})$ that a given configuration $\mathbf{s}$ occurs at temperature $T$ is proportional to the Boltzmann canonical

\footnotetext{
*buonsante@polito.it

†burioni@fis.unipr.it

$¥$ cassi@fis.unipr.it

$\S$ vezzani@fis.unipr.it
}

factor, $\exp \left[-T^{-1} E(\mathbf{s})\right]$, independent of the initial configuration.

One of the most commonly exploited algorithms is the Glauber's single-spin-flip algorithmE. According to Glauber's prescription two subsequent configurations can differ at most by the value of a single spin variable. More precisely the spin of the configuration $\mathbf{s}$ relevant to the site $k$ can flip with probability

$$
p_{T}^{\mathrm{G}}(\mathbf{s}, k)=\frac{1}{1+\exp \left[T^{-1} \Delta E_{k}(\mathbf{s}),\right]}
$$

where

$$
\Delta E_{k}(\mathbf{s})=2 s_{k} \sum_{i \sim k} s_{i}
$$

is the energy variation consequent to the process. Glauber's dynamics is completely defined by Eq. (2) together with a prescription for updating the spin system. The latter is commonly chosen in view of a computational optimization and typically copsists in an unphysical sweep along parallel lattice lines 6 .

As we mentioned above such a dynamics has a computational rather than physical origin, having been devised as an efficient way to give estimates of the canonical averages. Nevertheless it is usually given a physical interpretation 1 : the energy variation consequent to the spin-flips realizing the evolution of the system occur due to the coupling of the spin degrees of freedom to a heatbath at temperature $T$.

In the last decade a number of new dynamics for the Ising model were introduced $8 \mathrm{~g}$, where the energy variations are typically required to occur uniformly throughout the whole sample.

Here we introduce an alternative dynamics exhibiting the diffusive behaviour of a random walk in an evolving landscape generated by the energy of the spin couplings 
in the real space. The spin-flips are induced by a random walker hopping across the sites of the underlying discrete structure. The motion of such a random walker is affected by the spin interaction, being biased towards those sites where a spin-flip is energetically more favorable. It should be remarked that a relaxation dynamics occurring under the action of random walkers was introduced by Creutz10, and subsequently exploited by many authors. However our walkers act quite differently from Creutz's demons. Indeed the latter diffuse freely, unbiased by the magnetic configuration of the system, and, in order to simulate the micro-canonical ensemble, they induce a spin flip only if they can afford it, according to the individual energy they are endowed with.

In fact our model in inspired by the physical behaviour of manganites11, such as $\mathrm{La}_{x} \mathrm{Ca}_{1-x} \mathrm{MnO}_{3}$, where the spin dynamics is determined by the presence of diffusing excitations interacting with the magnetic degrees of freedom. In particular, for the manganites the excitations are given by the charged carriers, electrons and vacancies, present in the non-stoichiometrical compounds. Obviously, this model is not intended to be a phenomenological description of such complex systems, but it aims to evidence the influence of diffusing excitation interacting with spins on the thermodynamic behaviour of the paradigmatic model of all magnetic phase transitions.

The analysis of the diffusive dynamics is carried out from the numerical point of view. All the simulations illustrated in the present paper refer to two-dimensional arrays of spins, so that the observed features of the statistical model can be compared to the analytically known results pertaining to the canonical Ising model.

The plan of the paper is as follows: in Section II we describe and briefly discuss the diffusive algorithm. Section III and IV are devoted to the analysis of the limit situation where the dynamics is realized by a single walker. In the former we provide numerical evidences that the magnetic system is driven to a thermodynamically wellbehaved steady state which differs from the canonical equilibrium state in a non trivial-manner. Since a magnetic phase transition is still observed, the consequent critical behaviour is analyzed in Section IV. The estimated critical exponents do not deviate significantly from the values pertaining to the canonical Ising model. In Section $\mathrm{Z}$ the results of simulations where the system evolves subject to more than a single walker are analyzed. Section VI consists in our conclusions.

\section{DIFFUSIVE THERMAL DYNAMICS}

As we mentioned in the previous section the relaxation dynamics we propose is realized by means of random walkers diffusing through the sites of the Ising system. The probability that a walker located at site $i$ hops on site $j$ and flips the relevant spin, where $j$ is one of the $2 d$ neighbours of $i$, is given by

$$
p_{T}(\mathbf{s}, i, j)=(2 d)^{-1} p_{T}^{\mathrm{G}}(\mathbf{s}, j)
$$

Hence, for any configuration $\mathbf{s}$ of the Ising system and for any value of the temperature $T$, the diffusion of the walker is biased towards those sites where a spin-flip is more likely to occur according to Glauber's probability, Eq. (2). Note that Equation (4) implicitly yields a probability

$$
p_{T}(\mathbf{s}, i, i)=1-(2 d)^{-1} \sum_{k \sim i} p_{T}^{\mathrm{G}}(\mathbf{s}, k)
$$

that the walker does not move. If this is the case the magnetic configuration of the system $\mathbf{s}$ remains unchanged as well. According to equations (仼) and (5) the evolution of the probability $P(\mathbf{s}, i, t)$ that, at time step $t$, the magnetic system is in the configuration $\mathbf{s}$ while the walker is located at site $i$, is governed by the master equation

$$
\begin{array}{r}
P_{T}(\mathbf{s}, i, t+1)-P_{T}(\mathbf{s}, i, t)= \\
(2 d)^{-1} \sum_{j \sim i}\left[P_{T}\left(\mathbf{s}_{i}^{\prime}, j, t\right) p_{T}^{\mathrm{G}}\left(\mathbf{s}_{i}^{\prime}, i\right)+\right. \\
\left.-P_{T}(\mathbf{s}, i, t) p_{T}^{\mathrm{G}}(\mathbf{s}, j)\right],
\end{array}
$$

where $\mathbf{s}_{k}^{\prime}$ is a shorthand notation for the configuration obtained flipping the spin $s_{k}$ of $\mathbf{s}$. Unfortunately the master equation approach is of very little use in analyzing the long-time behaviour of this process. Actually in the canonical heat-bath case the master equation was exploited to build a dynamics driving the system to a known asymptotic probability. This was obtained by imposing the quite restrictive detailed balance condition. Conversely, we introduced an evolution algorithm and our aim is the study of the resulting asymptotic state of the Ising system, if any. Hence we carry out our analysis mainly by means of numerical simulations. For a better comparison we focus on the two-dimensional system, where most of the results pertaining to the canonical Ising model are exactly known. Hence all of our simulations refer to an Ising system consisting of $N=L^{2}$ spins placed at the sites of a two-dimensional square lattice. In order to avoid time consuming procedures dealing with the walkers bumping into the borders of the system we adopt periodic boundary conditions.

In the next two Sections we focus on the situation where the evolution of the Ising system is realized by a single walker. This is a limit case, in that, for a reasonably macroscopic system, it corresponds to a vanishing density of walkers. The results pertaining to larger densities are discussed in Section V.

\section{NON-CANONICAL EQUILIBRIUM STATES}

In the following we give numerical evidence that the spin-flip dynamics realized by a single walker drives the magnetic system to a thermodynamically well-behaved steady state which differs from the canonical equilibrium state in a non trivial manner. All the simulations we performed show that, at a given value of the external parameter $T$ the system eventually reaches a steady state, 


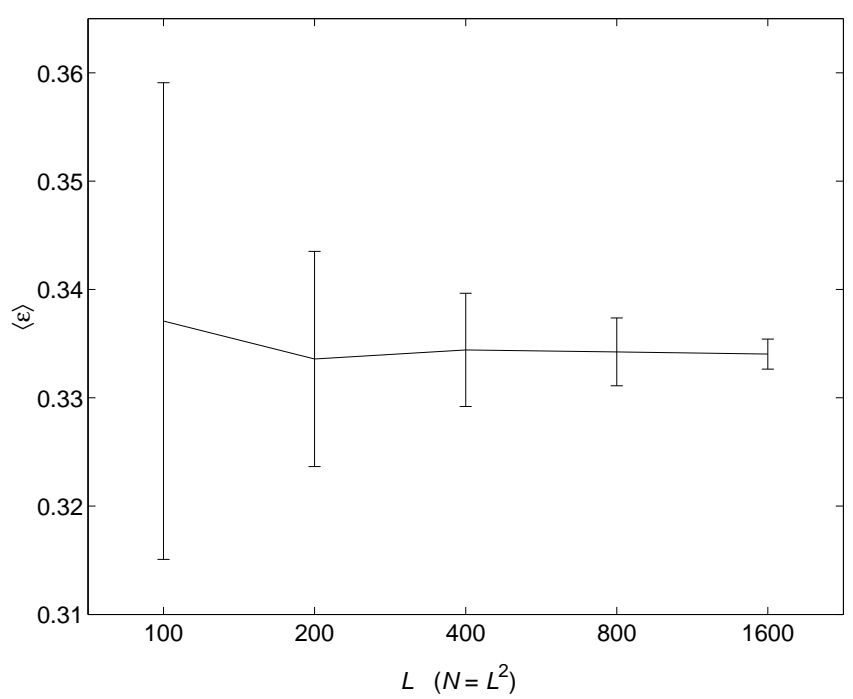

FIG. 1: Finite size scaling for the specific energy of an Ising system subject to the diffusive dynamics described in Section II at $T=2.4$. All the measurements were carried out in the stationary regime. The error-bars represent the fluctuations about the average values.

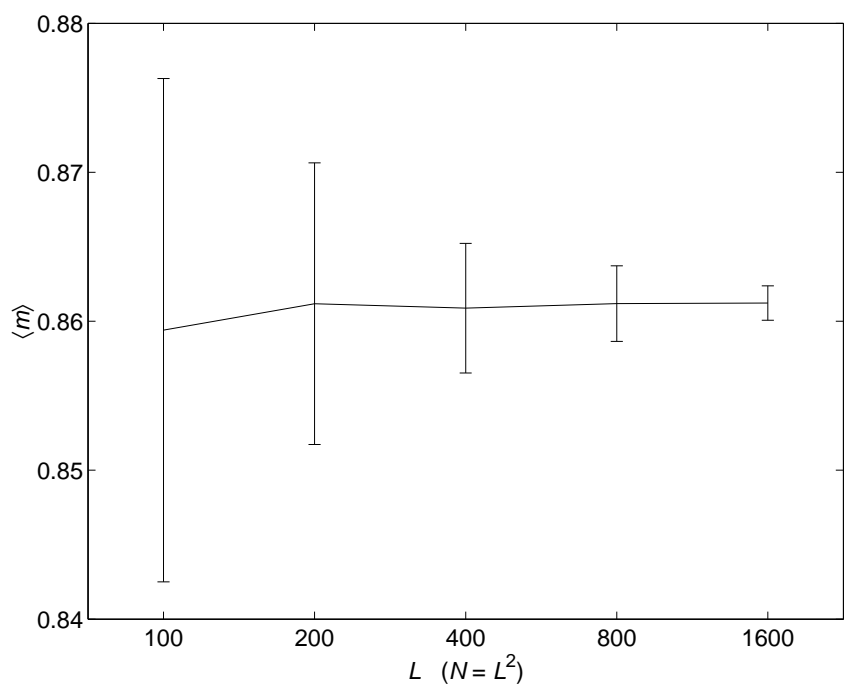

FIG. 2: Finite size scaling for the specific magnetization of an Ising system subject to the diffusive dynamics described in Section II at $T=2.4$. All the measurements were carried out in the stationary regime. The error-bars represent the fluctuations about the average values. Note that despite the symmetry of the Hamiltonian the measured value for the specific magnetization is non-zero. Similar to the canonical case the Ising system displays a phase transition.

characterized by a well defined value of the (time) average of the macroscopic observables. These features are clearly recognizable in Figures 1 and 2, where the average values of the specific energy $\epsilon(\mathbf{s})=N^{-1} E(\mathbf{s})$ and magnetization $m(\mathbf{s})=N^{-1} \sum_{i=1}^{N} s_{i}$ are plotted for systems with different sizes, at a fixed value of the temperature parameter. Furthermore, as one would require, the entire sample and any reasonably macroscopic portion of it are characterized by the same specific values of the thermodynamic observables. As it is clearly shown in Figures 3 and 4 , the fluctuations about the average value of the specific thermodynamic observables exhibit the expected scaling behaviour, decreasing as the inverse square root of the system size. Figure 5 shows the average values of

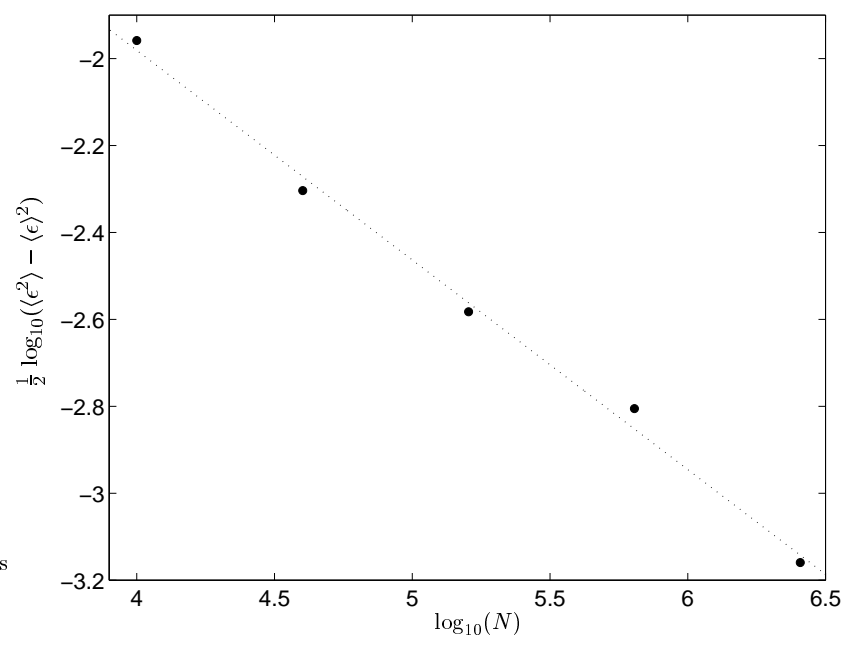

FIG. 3: Finite size scaling for the fluctuation about the average value of the specific energy for an Ising system subject to the diffusive dynamics described in Section II at $T=2.4$. The slope of the linear fit (dotted line) of the measured data $(\bullet)$, $-0.48 \pm 0.02$, is in good agreement with the expected value, 0.5. All the measurements were carried out in the stationary regime.

the specific energy and magnetization at different values of the temperature parameter, $T=\beta^{-1}$. Note that, similar to the canonical Ising model, the system exhibits a magnetic phase transition. We verified that the long time behaviour of the macroscopic observables of the system is uniquely determined by the only external parameter characterizing the dynamics, namely the temperature $T$. In particular different choices for the initial configuration of the magnetic system do not affect the asymptotic behaviour of the system, except for a possible trivial bias on the orientation of the spontaneous magnetization. Similar to what happens in the heat-bath case, both of the possible orientation of the specific magnetization are in principle equally likely, but a strongly magnetized initial configuration is very likely to evolve in a stationary state exhibiting a spontaneous magnetization along the same direction. When the initial configuration has no net magnetization there is no bias on the direction of the spontaneous magnetization exhibited by the system below the critical temperature. In this situations the formation of macroscopic domains exhibiting opposite net magnetization is observed (see Fig.6). In the long time regime one of the domains eventually prevails against the others. 


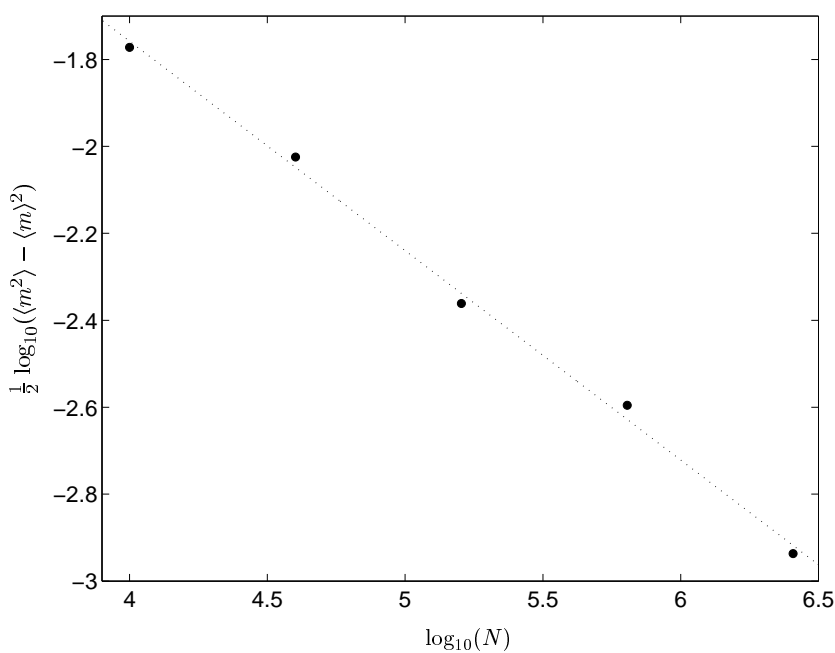

FIG. 4: Finite size scaling for the fluctuation about the average value of the specific magnetization for an Ising system subject to the diffusive dynamics described in Section II at $T=2.4$. The slope of the linear fit (dotted line) of the measured data $(\bullet),-0.48 \pm 0.02$, is in good agreement with the expected value, 0.5. All the measurements were carried out in the stationary regime.

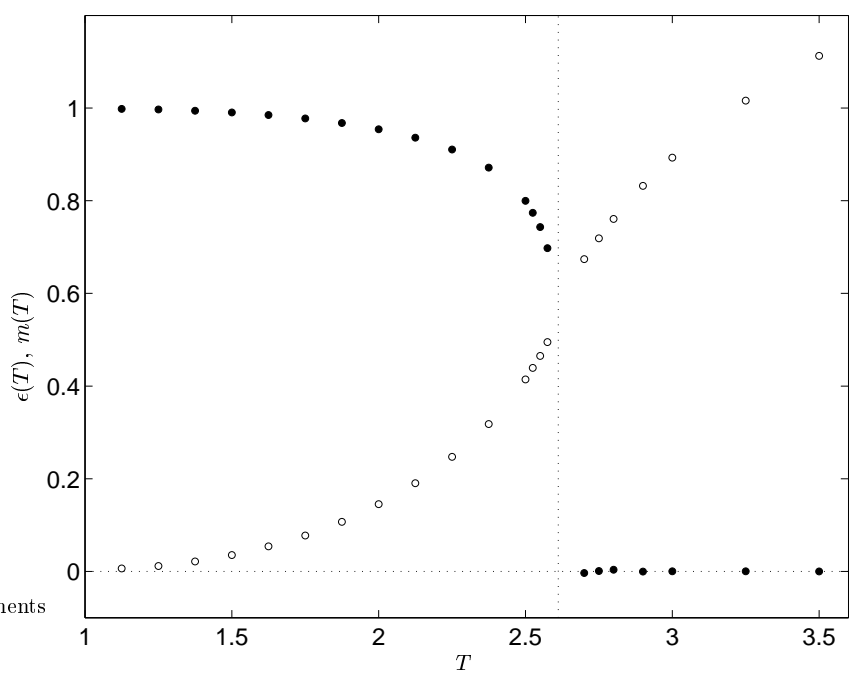

FIG. 5: Macroscopic observables for a $400 \times 400$ Ising array. Filled circles $(\bullet)$ : specific magnetization; open circles (o): specific energy.

As we already mentioned the system exhibits a magnetic phase transition. From Fig. 5 and 6, it is clear that the critical temperature for the diffusive dynamics is significantly larger than the value pertaining to the canonical Ising model. Such value, $T_{\mathrm{c}}^{\mathrm{Ising}}=2 / \ln (1+\sqrt{2}) \approx 2.269$ is analytically known and the heat-bath dynamics yield quite satisfactory numerical estimates of it. As we will see in the next Section, accurate estimates yield the value $T_{\mathrm{c}}=2.612 \pm 0.002$ for the critical temperature of an Ising

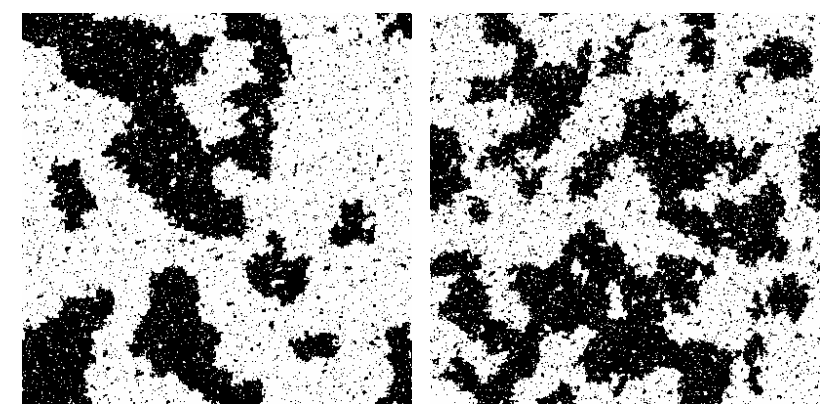

FIG. 6: Typical configurations for a $400 \times 400$ Ising array with magnetization $\langle m\rangle=0.86$ for the diffusive dynamics (left panel) and the Glauber's dynamics (right panel). Note that the same magnetization is attained for different temperatures. More precisely $T^{\text {diff }}=2.4>T^{\text {Ising }}$ and $T^{\text {Glauber }}=2.13<$ $T^{\text {Ising }}$, where $T_{\mathrm{c}}^{\text {Ising }}=2 / \ln (1+\sqrt{2}) \approx 2.269$ is the critical temperature of the canonical Ising model. Note further that in the diffusive case the domains present smoother boundaries.

system evolving under the action of a single walker. However, such a quantitative difference cannot be accounted for by means of a simple rescaling of the temperature. Indeed if the diffusive dynamics acted as heat-bath dynamics with a rescaled temperature, $T^{\prime}=\tau(T)$, the generic configuration s would occur with a probability $P(\mathbf{s}, T) \propto \exp \left[-T^{\prime-1} E(\mathbf{s})\right]$ in the asymptotic regime $\frac{1}{\text {. }}$. Hence the joint probability for the occurrence of a configuration such that $\epsilon(\mathbf{s})=\epsilon^{\prime}$ and $m(\mathbf{s})=m^{\prime}$ would be of the form

$$
\mathcal{P}\left(\epsilon^{\prime}, m^{\prime}, T\right)=Z(T) J\left(\epsilon^{\prime}, m^{\prime}\right) e^{-T^{\prime-1} N \epsilon^{\prime}},
$$

where

$$
J\left(\epsilon^{\prime}, m^{\prime}\right)=\sum_{\mathbf{s}} \delta\left(\epsilon^{\prime}-\epsilon(\mathbf{s})\right) \delta\left(m^{\prime}-m(\mathbf{s})\right)
$$

and

$$
Z(T)=\sum_{m^{\prime} \epsilon^{\prime}} J\left(\epsilon^{\prime}, m^{\prime}\right) e^{-T^{\prime-1} N \epsilon^{\prime}}=\sum_{\mathbf{s}} e^{-T^{\prime-1} E(\mathbf{s})}
$$

Last equality of Eq. (9) was obtained making use of Eq. (8). Now note that, according to Eqs. (7) and (8),

$$
\tilde{\mathcal{P}}(\epsilon, m, T) \equiv \frac{\mathcal{P}(\epsilon, m, T)}{\sum_{m} \mathcal{P}(\epsilon, m, T)}=\frac{J(\epsilon, m)}{\sum_{m} J(\epsilon, m)}
$$

Hence, if the rescaling hypothesis was true, the plot of $\tilde{\mathcal{P}}(\epsilon, m, T)$ versus $m$ at a fixed value of the specific energy would not depend on the temperature: the curves pertaining to the same specific energy at different values of the temperature $T$ would overlap. This can be verified with great precision in the case of the heat-bath dynamics 12 The numeric estimates of these curves for two different values of the specific energy $\epsilon$ are plotted in figures 7 and 8. The fact that the curves pertaining to the 


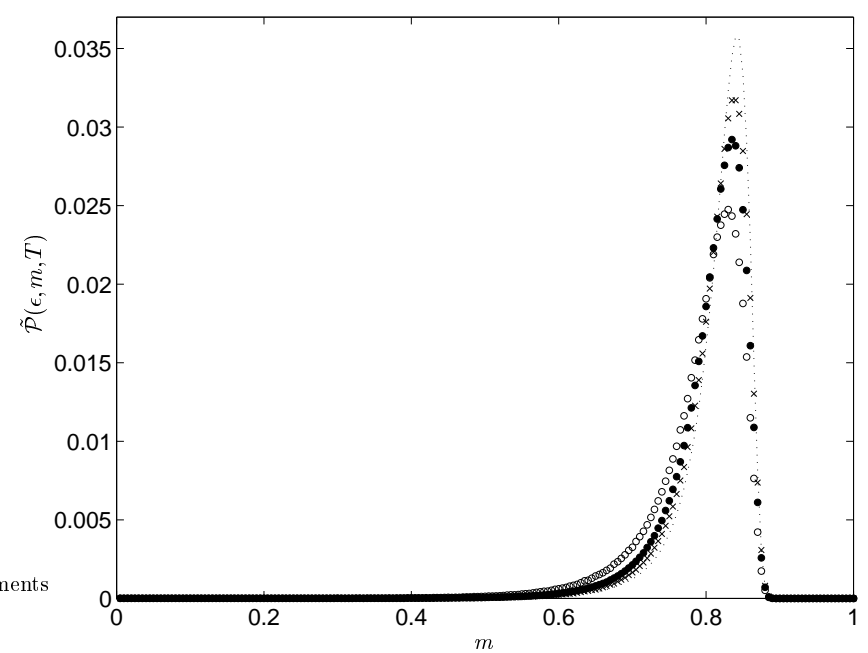

FIG. 7: Normalized joint distributions $\tilde{\mathcal{P}}(\epsilon, m, T)$ for a $20 \times 20$ Ising array subject to the diffusive dynamics. All of the curves refer to the specific energy $\epsilon=0.220$. Filled circles $(\bullet): T=$ 2.25; open circles (०): $T=2.50$; crosses $(\times): T=2.75$; dotted line: heat-bath dynamics (temperature independent).

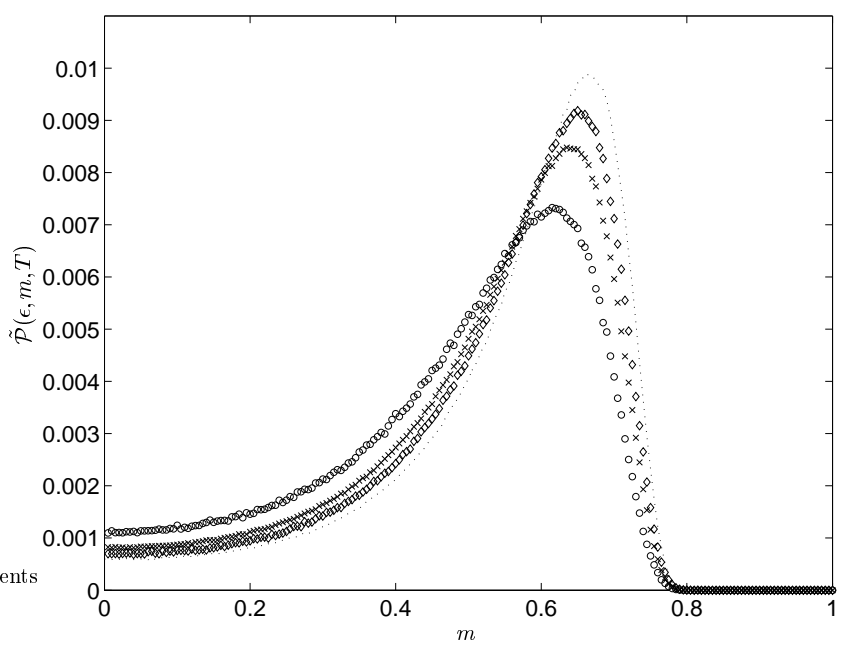

FIG. 8: Normalized joint distributions $\tilde{\mathcal{P}}(\epsilon, m, T)$ for a $20 \times 20$ Ising array subject to the diffusive dynamics. All of the curves refer to the specific energy $\epsilon=0.345$. Filled circles $(\bullet): T=$ 2.50; open circles (o): $T=2.75$; crosses $(\times): T=3.00$; dotted line: heat-bath dynamics (temperature independent).

diffusive dynamics at different temperatures are well distinct from one another and from the (temperature independent) curve characterizing the canonical Ising model proves that the differences between the diffusive and the heat-bath dynamics cannot be accounted for by means of a simple rescaling of the temperature.

In summary the steady state asymptotically reached by an Ising system subject to the action of the diffusive dynamics is thermodynamically well-behaved, and yet it is non-trivially different from the canonical equilibrium of the Ising model. Hence we will refer to the steady state of the diffusive dynamics as a non-canonical equilibrium state.

In the following section we analyze the critical behaviour of the Ising system subject to the diffusive dynamics.

\section{CRITICAL BEHAVIOUR}

As we already observed in the previous Section, an Ising system subject to a single diffusive walker exhibits a magnetic phase transition at a finite value of the temperature (see Figure 5). Similar to what happens with the Ising model, this phenomenon is accompanied by a singular behaviour of the thermodynamic functions. In

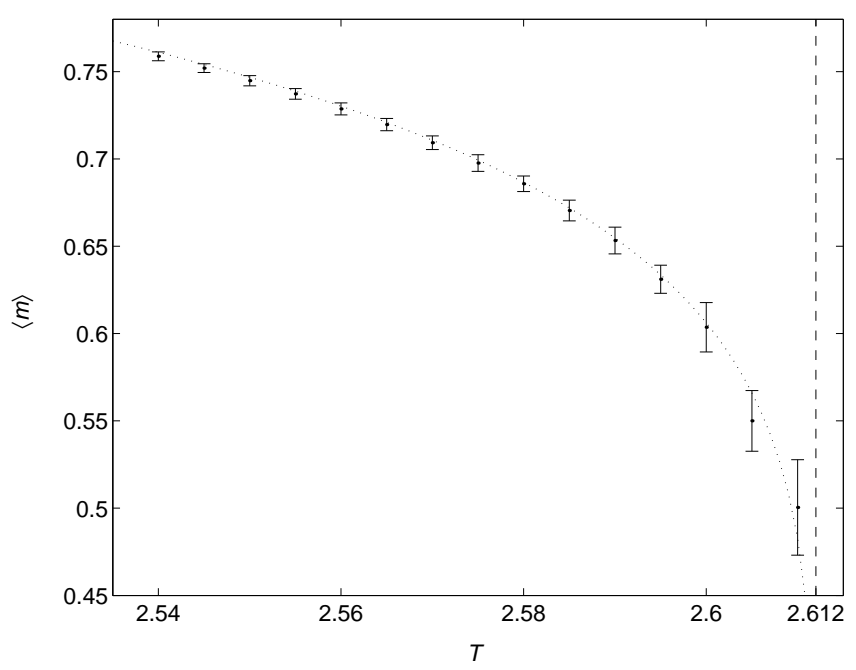

FIG. 9: Critical exponent, $\beta$, of the magnetization for an Ising system subject to the diffusive dynamics. The measures were performed on a system consisting of $N=2000 \times 2000$ spins. The error bars denote the standard deviation of the measures. The dotted line is the best fit, $y=A\left|T-T_{\mathrm{c}}\right|^{\beta}$. The estimated values for the critical temperature and for the exponent are $T_{\mathrm{c}}=2.612 \pm 0.001$ and $\beta=0.127 \pm 0.002$, respectively. The latter is consistent with the relevant critical exponent of the canonical Ising model, $\beta_{\text {Ising }}=1 / 8$. The vertical dashed line indicates the critical temperature.

Figure 9 accurate data for the magnetization of an Ising system subject to the diffusive dynamics are plotted. The error bars represent the standard deviations about the average values. Similar to the canonical case, the data show a good agreement with a critical behaviour of the form $m(T) \sim\left|T-T_{\mathrm{c}}\right|^{\beta}$, where the estimated values are for the critical temperature and for the critical exponent are $T_{\mathrm{c}}=2.612 \pm 0.001$ and $\beta=0.127 \pm 0.02$, respectively. The latter result is in good agreement with the relevant critical exponent of the two-dimensional canonical Ising model, $\beta_{\text {Ising }}=1 / 8$. As we already discussed in the previ- 
ous Section, the critical temperature is appreciably larger than the canonical value, $T_{\mathrm{c}}^{\text {Ising }} \approx 2.269$.

Figure 10 shows the specific heat $c(T)=d\langle\epsilon\rangle_{T} / d T$ for a $400 \times 400$ Ising array subject to the diffusive dynamics as a function of the temperature $T$. The vertical dashed lines are placed at the critical value of the temperature. The dotted curves refer to functions of the form $f(T)=a+b \log \left(\left|T-T_{\mathrm{c}}\right|\right)$, and they fit quite satisfactorily the data. Hence, similar to the canonical case, there is a strong signature of a logarithmic divergence of the specific heat at the critical temperature. Figure 11 shows

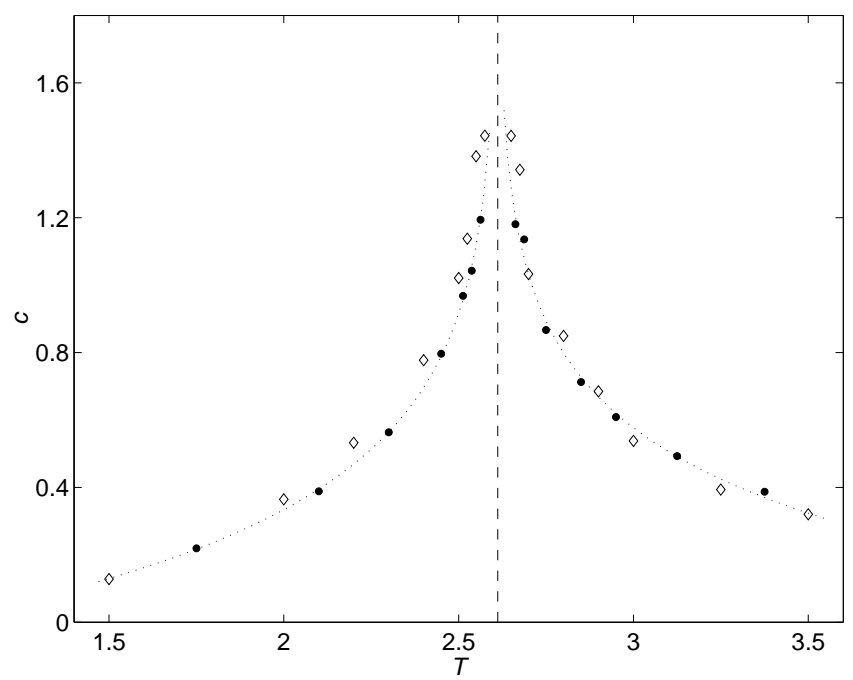

FIG. 10: Specific heat for an Ising system subject to diffusive dynamics $(\bullet)$. The dotted curves fitting the data are of the form $f(T)=a+b \log \left(\left|T-T_{\mathrm{c}}\right|\right)$. The vertical dashed line indicates the estimated value of the critical temperature, $T_{\mathrm{c}}=$ $2.612 \pm 0.002$. The diamonds $(\diamond)$ refer to the quantity $c_{\mathrm{f}}(T)$ defined in Eq. (12).

a $\log$-log scale plot of the quantity

$$
\chi_{\mathrm{f}}(T) \equiv \frac{N}{T}\left[\left\langle m^{2}\right\rangle_{T}-\langle m\rangle_{T}^{2}\right]
$$

versus $\left|T-T_{\mathrm{c}}\right|$. The data are consistent with a power law of the form $\chi_{\mathrm{f}}(T) \sim\left|T-T_{\mathrm{c}}\right|^{\gamma}$, and the slope $\gamma=1.73 \pm 0.06$ of the linear fit is in good agreement with the critical exponent governing the behaviour of the same quantity in the of the canonical case, $\gamma_{\text {Ising }}=7 / 4$. We recall that for a system at canonical equilibrium the fluctuation-dissipation theorem yields the relations $c(T)=c_{\mathrm{f}}(T)$ and $\chi(T)=\chi_{\mathrm{f}}(T)$, where

$$
c_{\mathrm{f}}(T)=\frac{N}{T^{2}}\left[\left\langle\epsilon^{2}\right\rangle_{T}-\langle\epsilon\rangle_{T}^{2}\right] .
$$

and $\chi(T)=\partial\langle m\rangle_{T, h} / \partial h$ is the magnetic susceptibility of the system. In our case this is not necessarily true, since the equilibrium distribution for a diffusive dynamics at the temperature $T$ is not proportional to the canonical Boltzmann factor exp $\left[-T^{-1} H\right]$, as we discussed in the previous section. Hence the critical behaviour of two quantities canonically related by a fluctuation-dissipation relation should be explicitly analyzed and compared. A preliminary step in this sense is shown in figure 10, where the diamonds represent the estimates of the quantity $c_{\mathrm{f}}(T)$ defined in Eq. (12). These results seem to indicate that the diffusive character of the dynamics does not produce large deviations from the fluctuation-dissipation relation. Of course this result needs to be checked through the analysis of the response of the system to an external magnetic field. However we mention that other studies 13 of Ising systems subject to dynamics which do not yield the canonical equilibrium state actually estimate the critical exponent $\gamma$ in terms of the quantity $\chi_{\mathrm{f}}(T)$ defined in Eq. (11).

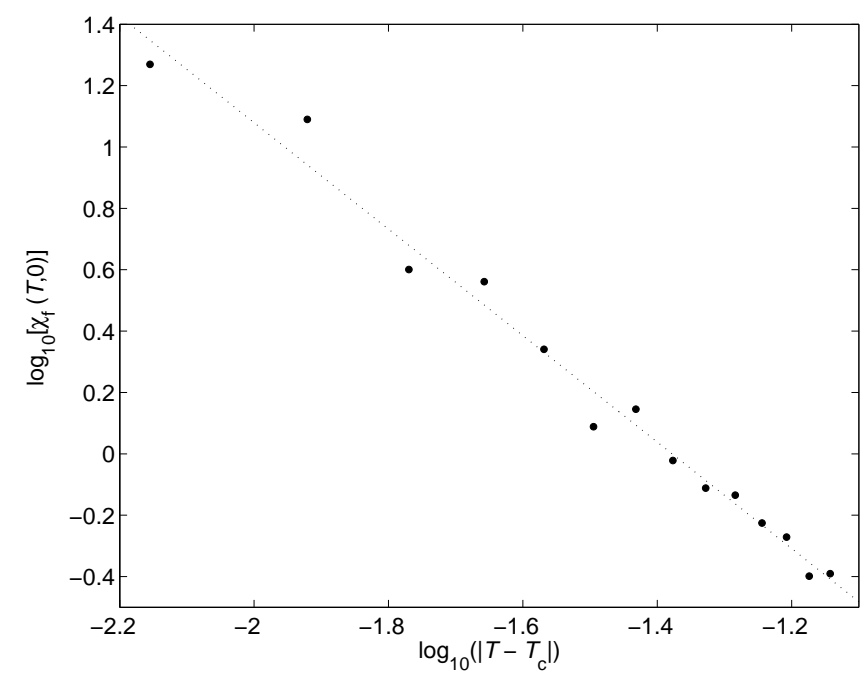

FIG. 11: Log-log scale plot of the magnetic susceptibility $\chi_{\mathrm{f}}$ (defined in terms of the magnetic fluctuations, see Eq. (11)) versus $\left|T-T_{\mathrm{c}}\right|$. The estimated slope of the straight line fitting the data is $-1.73 \pm 0.07$. This value is consistent with the critical behaviour of the magnetic susceptibility for the two-dimensional canonical Ising model, characterized by the critical exponent $\gamma_{\text {Ising }}=7 / 4$.

For temperatures sufficiently far from the critical value the correlation function

$$
\Gamma_{T}(i, j)=\left\langle s_{i} s_{j}\right\rangle_{T}-\left\langle s_{i}\right\rangle_{T}\left\langle s_{j}\right\rangle_{T}
$$

exhibits an exponential decrease as the distance between the relevant sites, $r_{i j}=\|i-j\|$, increases:

$$
\Gamma_{T}(i, j) \propto \exp \left[-\frac{r_{i j}}{\xi(T)}\right]
$$

As it is shown in Fig. 12 the correlation distance $\xi(T)$ features a maximum very close to the critical temperature. The fact that this maximum suggests a critical temperature slightly larger than the value previously (and more accurately) estimated must be ascribed to finite size effects. We further mention that the correlation lengths 
are roughly consistent with a power-law divergence of the form $\xi(T) \sim\left|T-T_{\mathrm{c}}\right|^{-\nu}$, the estimated exponent being remarkably close to the relevant critical exponent of the canonical two-dimensional Ising model, $\nu_{\text {Ising }}=1$. As it

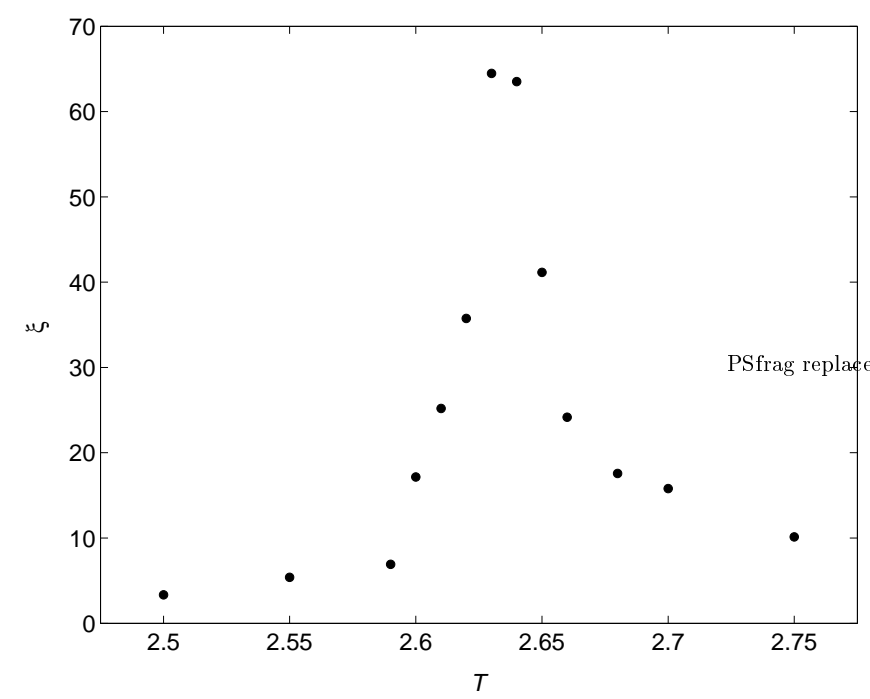

FIG. 12: Correlation length as a function of the temperature for a $600 \times 600$ Ising array subject to the diffusive dynamics. Due to finite size effects, the critical behaviour is exhibited for temperature slightly higher than the estimated critical value, $T_{\mathrm{c}}=2.612 \pm 0.002$.

is well known 14, for temperatures very close to the critical value, and hence for correlation lengths very large compared to the lattice constant, the correlation function of the canonical Ising model is characterized by a power-law behaviour of the form $\Gamma_{T}(i, j) \propto r_{i j}^{p}$. The relevant critical exponent is defined to be $\eta=p-d+2$, where $d$ is the dimensionality of the Ising array. For the two-dimensional canonical Ising model its value is exactly known to be $\eta_{\text {Ising }}=1 / 4$. Such behaviour is expected to be observable in the distance range $1 \ll r_{1 j} \ll \xi(T)$. As it is shown in Figure 13 the data yielding the largest correlation length are not consistent with a pure power law behaviour, not even for relatively small distances. This suggests that the relevant temperatures, $T=2.63$ is not sufficiently close to the critical value. Moreover it should be recalled that the finite size of the system can introduce appreciable deviations from the theoretical prediction, which is strictly true in the thermodynamic limit. Nevertheless we mention that a function of the form

$$
f(r)=a \exp \left(-\frac{r}{\xi(T)}\right) r^{-\eta}
$$

fits the data quite satisfactorily over a wide range of distances. The estimate for the exponent, $\eta=0.252 \pm 0.002$ is remarkably close to the critical exponent $\eta_{\text {Ising }}$ of the two-dimensional Ising model. A fit of the same kind satisfactorily applies to the data relevant to the temperature $T=2.64$, and it gives a critical exponent

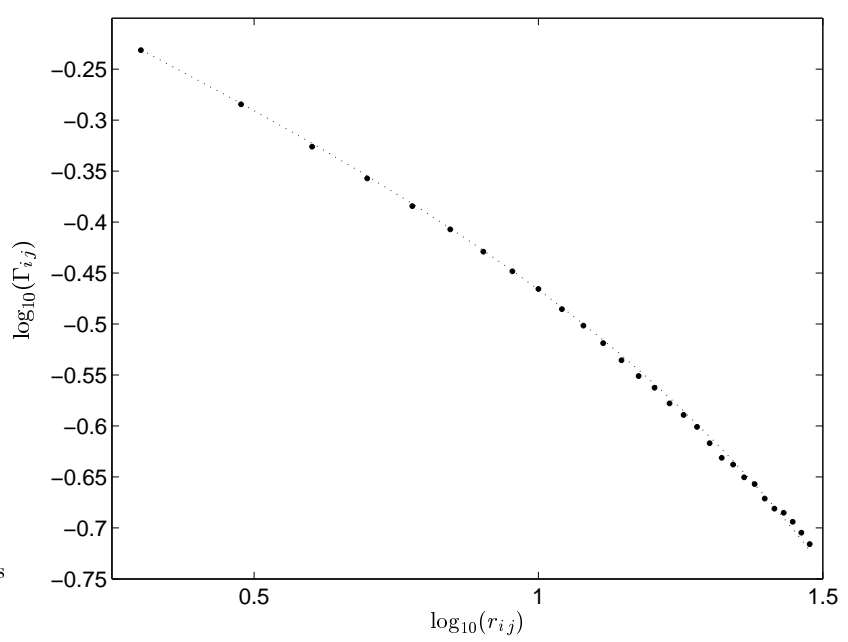

FIG. 13: Log-log scale plot of the correlation function $\Gamma_{T=2.63}(i, j)$ versus the inter-spin distance, $r_{i j}$. The data appreciably deviate from a power law behaviour. The dotted line refer to a function of the form defined by Eq. (15). The exponent $\eta$ is quite close to the critical exponent $\eta_{\text {Ising }}=1 / 4$. More precisely we estimate $\eta_{2.63}=0.252 \pm 0.002$. The same fit satisfactorily applies to the data relevant to $T=2.64$, where the exponent $\eta_{2.64}=0.266 \pm 0.003$ is once again in good agreement with the canonical value.

$\eta=0.266 \pm 0.002$ once again in good agreement with the canonical value. These results could be an indication of the fact that, in the suitable regime, the correlation function $\Gamma_{T \approx T_{\mathrm{c}}}(i j)$ actually exhibits a power law behaviour characterized by the same critical exponent as the two-dimensional canonical Ising model.

The results illustrated so far suggest that a twodimensional Ising ferromagnet subject to the diffusive dynamics exhibits a critical behaviour belonging to the same universality class as the canonical case.

Before concluding the present section we mention a further result which has no counterpart in the canonical case. Indeed, in the diffusive case, it is possible to relate the configuration of the magnetic system to the position of the random walker causing its evolution. More precisely it is possible to measure to what extent the presence of the random walker at a given site $i$ and the local magnetization at a given site $j$ influence each other. $\mathrm{Nu}-$ merical estimates of such correlation, which we illustrate elsewhere 15 , show that it exhibits a critical behaviour in correspondence of the critical temperature.

\section{RECOVERING THE CANONICAL EQUILIBRIUM: DENSITY OF WALKERS}

The results illustrated in the previous two Sections refer to the case of an Ising system subject to the action of a single walker. Here we analyze the results of simulations where the evolution of a square Ising array consisting of 
$N$ spins is realized by $n$ non interacting walkers. The simulation is initialized giving each walker a randomly chosen position. Two or more walkers are allowed to occupy the same site of the system. Actually this is very likely to happen for a sufficiently large density of walkers $\rho=n / N$. An elementary step of the simulation consists in the application of the algorithm described in Section II to all of the walkers, according to a fixed progressive sequence. When its turn comes, a given walker acts as if the other ones were not present. A qualitative argument leads us to expect that in the case of an infinite density of walkers the results pertaining to the canonical Ising model are recovered. Consider a very large density of walkers, such that $n \gg N$. In this situation the effect of a single step of the simulation is very similar to the action of the Glauber's dynamics with a random sequence update. Indeed the probability that a walker flips a given spin $s_{i}$ is given by the product of the probability $4 / N$ that it is located at one of the four neighbours of site $i$ times the hopping probability, $p^{\mathrm{G}}(\mathbf{s}, i) / 4$. But $p^{\mathrm{G}}(\mathbf{s}, i) / N$ is exactly the probability that the spin $s_{i}$ is flipped at any step of the Glauber's heat-bath dynamics with random site update. Hence, in the limit case of an infinite density of walkers, the diffusive dynamics should necessarily drive the system to the same asymptotic state as the heat-bath dynamics, thus reproducing the results of the canonical Ising model. The same should be clearly true for a sufficiently large density of walkers, provided that the consequent number of elementary steps of the heat-bath dynamics drive the system sufficiently close to the canonical equilibrium state. The numerical simula-

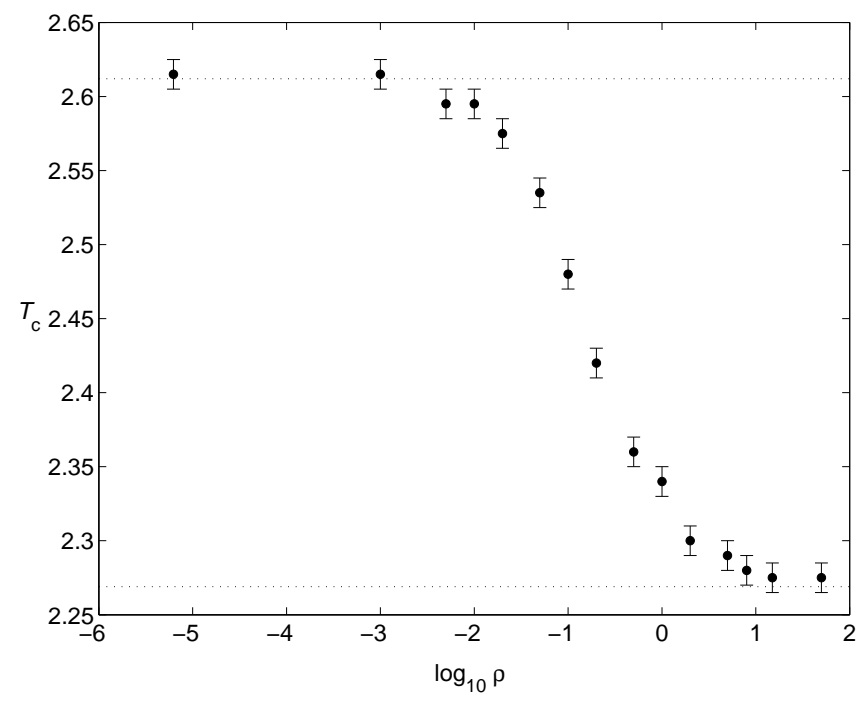

FIG. 14: Critical temperatures with increasing density of walkers for a $400 \times 400$ Ising array. The horizontal dotted lines are placed at the critical temperatures of the diffusive dynamics with a single walker $\left(T_{\mathrm{c}} \approx 2.612\right)$ and at the critical temperature of the canonical Ising model $\left(T_{\mathrm{c}}^{\mathrm{Ising}} \approx 2.269\right)$.

tions we performed evidenced that the diffusive dynamics drives the Ising system to a thermodynamically well behaved stationary state, so that the average values of the specific energy and magnetization are characterized only by the temperature, $T$, and the density of walkers, $\rho$. In particular they confirmed the qualitative argument discussed above: the results pertaining to the canonical Ising model at a given temperature $T$ were recovered for a sufficiently large density of walkers. More generally we verified that the average value of a macroscopic observable relevant to a finite density lies within the interval between the values pertaining to the limit situations, namely the vanishing density limit $\rho=N^{-1} \sim 0$ analyzed in Sections [II] and IV, and the canonical Ising model. The influence of the density of walkers is synthesized in Figure 14, where the estimated values of the critical temperature are plotted. Note that the larger variation occurs for densities between $10^{-2}$ and 1 .

\section{CONCLUSIONS AND PERSPECTIVES}

In this paper we exploited the basic microscopic model of diffusion, namely the random walk, to build a dynamics for the Ising ferromagnet which retains the diffusive character of the thermal motion of a localized excitation. In this framework the density of walkers is related both to the intensity and spatial extent of the coupling of the spins to the thermal degrees of freedom of the system, and hence to the velocity of the thermal dynamics. The larger is the density of walkers, the faster is the dynamics and the closer to the uniform heath-bath are its effects.

Even if our model is rather simplified with respect to the real physical systems which inspired it, it exhibits new important qualitative features which should be relevant even in more complex and realistic situations. Therefore it would be of great interest to verify whether the diffusive thermal dynamics gives a satisfactory description for the slow evolution of a magnetic system with hopping excitations. This would of course require the extension of the study of its effect on the more realistic three dimensional lattice. In view of recognizing the action of a diffusive dynamics in a real system, the results of Section IV suggest that the study of the critical behaviour is not particularly significant. Indeed the latter does not allow to discriminate among the canonical and the diffusive dynamics.

On the other hand the results illustrated in section $\mathrm{V}$ indicate that the velocity of the non-equilibrium dynamics (related to the degree of coupling of the spins to the thermal vibrations, i.e. to the density of excitations induced in the system by an external source) significantly affects the critical temperature of the system. Hence the same system subject to diffusive thermal dynamics with different velocity should display different critical temperatures. Furthermore we remark that Fig. 6 suggests that the velocity of the dynamics influences the shape and growth of the macroscopic magnetic domains.

In particular a very slow diffusive dynamics gives rise to domains with smoother boundaries. This feature sug- 
gests that the final shape of the magnetic domains within a physical system possibly governed by a diffusive thermal dynamics could be controlled by a fine tuning of the density of excitations mediating the coupling to the thermal degrees of freedom. A detailed study of domain growth in the diffysive dynamics will be the subject of a forthcoming paper 1 .

\section{Acknowledgments}

We are grateful to R. De Renzi for useful discussions.
1 E. Ising, Z. Phys. 31, 253 (1925).

2 L. Onsager, Phys. Rev. 65, 117 (1944).

3 B. M. McCoy and T. T. Wu, The Two Dimensional Ising Model, Harvard University Press, 1973.

${ }^{4}$ K. Binder, editor, Monte Carlo Methods in Statistical Physics, Springer-Verlag, 1986.

5 R. J. Glauber, J. Math. Phys. 4, 294 (1963).

6 This prescription is often referred to as typewriter update sequence. On a bipartite lattice it may prove numerically efficient to update each sublattice according to a typewriter sequence. The resulting sequence is referred to as checkerboard update sequence. See e.g. T. Vojta and M. Schreiber, Phys. Rev. E, 58, 7998 (1998).

7 See e.g A.M. Mariz, F. Nobre and C. Tsallis, Phys. Rev.
B 493576 (1994) and references therein.

8 J.S. Wang, Eur. Phys. J. B 8, 287 (1999).

9 J.R. Heringa and W.J. Blöte, Phys. Rev. E 574976 (1998).

10 M. Creutz, Phys. Rev. Lett. 50, 1411 (1983); G. Bhanot, M. Creutz and H. Neuberger, Nucl. Phys B 235, 417 (1984)

11 E. Dagotto, T. Hotta and A. Moreo, Phys. Rep. 344, 1 (2001).

12 In that case the rescaling is trivial: $\tau(x)=x$.

13 H. W. Blöte, J. R. Heringa, A. Hoogland, and R. K. P. Zia, J. Phys. A: Math. Gen. 23, 3799 (1990).

14 L. P. Kadanoff, Il Nuovo Cimento B, XLIV, 5152 (1966).

15 P. Buonsante, R. Burioni, D. Cassi and A. Vezzani, in preparation. 